\title{
Estimating Numbers and Survival of House Flies (Diptera: Muscidae) with Mark/Recapture Methods
}

\author{
T. J. LYSYK ${ }^{1}$ AND R. C. AXTELL \\ Department of Entomology, North Carolina State University, \\ Raleigh, North Carolina 27695-7613
}

J. Econ. Entomol. 79: 1016-1022 (1986)

\begin{abstract}
Two applications of the Jolly-Seber model were used to estimate numbers and survival of house flies, Musca domestica L. The multisample, single-recapture census, using reared, marked flies and capture by baited jug-traps, was used to estimate numbers of adult house flies in a two-story, high-rise, caged-layer poultry house. Density estimates ranged from 1.2 to 11.5 flies per $\mathrm{m}^{2}$ and had coefficients of variation of $0.12-0.52$. Daily survival rates (proportion of population which did not die or emigrate during the interval between samples) were 0.19-0.66 per day. The multisample, multirecapture census, using marked native flies and capture by netting, was used to estimate numbers and survival of house flies in a screen-sided, narrow caged-layer poultry house. Estimated fly densities ranged from 24.8 to $1,159.6$ flies per $\mathrm{m}^{2}$ with coefficients of variation of $0.03-0.48$. Daily survival rates were $0.54-0.99$ per day. Survival rates in both studies declined with increasing mean daily temperature $\left({ }^{\circ} \mathrm{C}\right)$ and on average were 0.32 lower in the high-rise house due to effects of dispersal and predation. House fly survival was not affected by marking with oilsoluble dyes, and capture of house flies using the baited jug-trap was not influenced by age, sex, or marked status of the flies. Assumptions in both census methods are discussed and shown to be met under conditions of these experiments.
\end{abstract}

KEY WORDS Musca domestica, mark/recapture, survival, populations, poultry houses

MARK/RECAPTURE METHODS are valuable tools for estimating numbers of mobile insects and have been applied to populations of the house fly, Musca domestica L. (Murvosh \& Thaggard 1966, Beck 1982, Pospisil \& Bogac 1982, McDonald et al. 1983, Imai 1984, Kristiansen \& Skovmand 1985). Several methods are available; each has its own set of assumptions that must be satisfied to obtain valid estimates of numbers. The Petersen model has been most frequently used for fly populations because it is simple to apply and requires only that a single sample be taken from a population into which a known number of marked flies have been released (Fletcher et al. 1981). Use of this model requires the assumptions that the population is closed during the sample period (i.e., no births, deaths, immigration, or emigration occur), all insects regardless of age, sex, or marked status have an equal probability of capture, and marks are permanent and recognizable (Pollock 1981).

The Petersen model is for closed populations or populations with either mortality or immigration, but not both. Its use with a house fly population, therefore, requires that the time between release and recapture of marked individuals be short relative to the life span of the insect (Southwood 1978). Intervals between release and recapture of marked flies of $24 \mathrm{~h}$ (Murvosh \& Thaggard 1966), 3-4 days (McDonald et al. 1983), and 7 days

${ }^{1}$ Current address: Canadian Forest Service, P.O. Box 490, Sault Ste. Marie, Ontario P6A 5M7, Canada.
(Pospisil \& Bogac 1982) have been used in applying this model to house fly populations. The long sampling intervals were used in those studies to obtain a large number of recaptures; however, the assumption of closure was likely violated as a result of the loss of marked individuals due to dispersal and mortality. The longer the interval, the greater the decline in the number of marked insects and the more biased the estimate of population numbers will be (Fletcher et al. 1981).

Seber (1962) described an open model that allows gains and losses for estimating survival and numbers of animals when releases of marked individuals are independent of recaptures and the loss on capture is $100 \%$. This situation, called the multisample, single-recapture census, is analogous to the case in which marked insects are reared from cultures, released into a population, and the population sampled with traps that kill the insect. Rather than a single release of marked insects, several releases of insects bearing date-specific marks are required. This method is useful in situations when live captures are impossible and traps have to be used. This model was later shown (Seber 1980 ) to be an extension of the well-known JollySeber model (Jolly 1965).

The assumptions underlying the Jolly-Seber model are every animal has the same probability of dying or permanently emigrating during each sampling interval, every animal has the same probability of capture, and there is no mark loss (Pollock 1981). The information gained from a se- 
ries of $n$ releases and recaptures are $n-2$ estimates of survival and numbers. The Jolly-Seber model has been used to estimate numbers and survival of the house fly in garbage dumps in Japan (Imai 1984). More recently, the Bailey (1952) triple catch method, which is a special case of the Jolly-Seber model (Begon 1979), was used to estimate numbers and survival of the house fly on livestock farms in Denmark (Kristiansen \& Skovmand 1985). Both studies were conducted on house fly populations that were accessible to the investigators and could be easily sampled with sweep nets. Since live captures were made on three or more sample occasions, and marked flies could be captured more than once, these studies provide an example of a census approach that we term the multisample, multirecapture census. This method is not always feasible for house fly populations in poultry production systems, particularly the highrise caged layer houses, because much of the fly habitat is inaccessible to the investigator due to the enormous quantities of accumulated manure present.

Two series of mark/recapture experiments were conducted in two poultry houses to demonstrate the suitability of the two census techniques for house fly population studies under field conditions. In addition, patterns of house fly abundance and survival in the presence and absence of dispersal were examined throughout the fly-breeding season.

\section{Materials and Methods}

Evaluation of Marking and Sampling Methods. Laboratory experiments were conducted to determine if the marking and sampling methods met the assumptions of the Jolly-Seber model. The possibility of heterogeneity in the capture of house flies with baited jug-traps (Burg \& Axtell 1984) due to age, sex, or marked status of the flies was investigated using screen cages $\left(1 \mathrm{~m}^{3}\right)$. The baited jug-trap was a plastic milk jug (3.5 liter) with four holes $(5 \mathrm{~cm}$ diam) cut in the side. The traps were baited with Improved Golden Malrin fly bait ( 25 g) containing $1.0 \%$ methomyl and $0.025 \%(Z)-9-$ tricosene (Zoecon, Dallas, Tex). The effect of age and sex of flies on capture with the trap was tested by releasing groups of 250 male and 250 female flies aged $1,3,5$, and 7 days into separate cages (with food and water) and sampling each cage for $24 \mathrm{~h}$ with a baited jug-trap. This procedure was replicated eight times, and the $\log _{10}$ of the number of flies of each age captured was used in a randomized complete block analysis of variance to determine if there were significant differences in capture due to age of the flies. The transformation was applied to stabilize the variances of the observations since these were counts. A $\chi^{2}$ test was used to determine if males and females were captured in equal proportions.

The effect of marking on capture was tested in a similar manner, except that 100 male and 100 female 3-day-old marked flies were released into cages already containing 3-day-old unmarked male and female flies (100 each), provided with food and water and sampled with a trap for $24 \mathrm{~h}$. Four cages were used for each dye, and a $\chi^{2}$ test was used to determine if marked and unmarked flies were caught in equal proportions.

Marking and detection methods were similar to those previously used on the house fly (Quarterman et al. 1954, Peffly \& LaBreque 1956, Lysyk \& Axtell 1986). Flies were marked $1 \mathrm{~h}$ before release by spraying cold-immobilized flies with a fine mist of a $2.5 \%$ solution of oil-soluble dye in acetone. The dyes were Calco red, Calco violet, Calco green (American Cyanamid, Bound Brook, N.J.), and phenolphthalein. The captured flies were examined for marks by placing them on white blotting paper and putting several drops of acetone on each. A colored ring formed around each marked fly corresponding to the dye used. Flies marked with phenolphthalein were detected by spraying a $1 \%$ solution of $\mathrm{NaOH}$ over the flies after the application of acetone and flies marked with this dye turned purple. Flies which showed no color were classified as unmarked.

The effect of dyes on survival of flies was tested by spraying five groups of flies per dye $(25$ male and 25 female flies per group), and holding the treated flies and five cages of 50 untreated flies as a control in screen-topped paper cylinders provisioned with food and water. After 5 days, the number of dead flies per 50 flies treated was counted, transformed to $\log _{10}$, and used in an analysis of variance to determine if there was an effect due to marking.

Multisample, Single-recapture Census. This study was conducted in a two-story, high-rise, caged-layer poultry house (Chatham County, N.C.) ca. $130 \mathrm{~m}$ long by $12 \mathrm{~m}$ wide. Birds were housed in the upper floor in eight rows of two-tiered cages (three birds per cage) separated by five walkways. The manure accumulated on the ground ca. $4 \mathrm{~m}$ below the cages.

A series of six 5-day trials of the multisample, single-recapture census (Seber 1962, Jolly 1965) was conducted from May to October 1983 to estimate daily survival rates and numbers of house flies. The house flies used for marking and releasing were reared from a colony started with wild flies collected from a poultry house in Wake County, N.C., the previous fall. Larvae were reared on fly rearing medium (Ralston Purina, St. Louis, Mo.)/yeast/water (CSMA medium). Pupae were separated from the medium with a forced air blower, and aliquot samples of 500 pupae were counted, weighed, and used to determine percent emergence. Depending on the number available, 10,000-11,000 pupae were weighed and placed into eight screen cages (11.6 liter) and held for emergence. Flies were held in the cages 1-2 days after emergence and fed a solution of milk and 
$10 \%$ sucrose in water. Rearing was timed so that 8,000-10,000 flies (1-2 days old) would be available for marking and release on each of 4 successive days of each series of releases. The number of flies released each day was determined by multiplying the number of pupae collected for the release by the proportion of the aliquot pupae that emerged.

Flies were marked $1 \mathrm{~h}$ before release and transported to the study site in an air-conditioned vehicle. Releases were made in the high-rise house before 0900 hours on day 1 by opening the cages slightly and carrying them slowly throughout the house to ensure mixture with the wild population. One hour after release, 24 baited jug-traps were placed in the house. Four traps were hung equally spaced from the rafters above each of the two outer walkways and four above the center walkway. Four equally spaced traps were also suspended 2 $\mathrm{m}$ below the walkway and ca. $2 \mathrm{~m}$ above the manure bed along each of the two outer walkways and below the center walkway. The traps were left in position until 0900 hours the next day (day 2), after which they were removed, the dead flies and bait removed from each trap, and fresh bait added. A fresh group of flies marked with a different dye was released in the house and the traps replaced $1 \mathrm{~h}$ later. This was repeated on days 3 and 4 ; on day 5 the traps were removed and no releases were made.

Temperature was recorded in the house at 3-h intervals by placing 12 temperature recorders in the rafters, each near the location of a trap; the average daily temperature was calculated for each day of the experiment. Flies captured each day were examined for marks in the laboratory and the number of unmarked and marked flies captured per trap per day was recorded. The data were analyzed by the method of Jolly (1965).

Multisample, Multirecapture Census. This experiment was conducted in a screen-sided (14 mesh), narrow caged-layer poultry house $(4.9 \mathrm{~m}$ long by $3.1 \mathrm{~m}$ wide) located in Wake County, N.C. The sides of the house were $2 \mathrm{~m}$ high and the roof rose to a central peak $3 \mathrm{~m}$ above the middle walkway. On each side of the walkway were two tiers of 10 standard commercial cages containing a total of 80 White Leghorn laying hens. Manure accumulated on the ground for 3 weeks before the beginning of the experiment and continued throughout (May-August 1984). The screens were left in place throughout the experiment and were cleaned periodically to remove debris and promote airflow. Spider webs were removed from the walls and rafters daily during sample periods.

Mark/recapture experiments were conducted in this house on 20-28 May, 13-17 and 26-28 June, $3-5,17-19$, and 24-26 July, 31 July-2 August, and 7-9 August. A 2-day interval between samples was used in the first two trials and flies were individually marked on the thorax with spots of enamel paint (Testors, Rockford, Ill.). This proved to be too time consuming and recapture of marked flies was so low in the second trial that in subsequent trials 1-day sampling intervals were used and flies were mass-marked with fluorescent powders (DayGlo, Cleveland, Ohio, and USR Optonix, Hackettstown, N.J.).

Flies were captured live with a sweep net. Sampling was uniformly along the walkways throughout the entire poultry house between $0800-0900$ hours. On the first sample date, flies were immobilized by chilling, and counted, dusted with a fluorescent powder (specific color for each date), and returned to the population. The different colored marks were detected on subsequent days by placing captured flies under an ultraviolet lamp. Each day the numbers of marked and unmarked flies in the sample were counted. Multiple captures were recorded. The data were analyzed with Jolly's (1965) method. Temperatures in the house were recorded at 3-h intervals with two temperature recorders placed in the rafters above the center aisle at each end of the house, and the average daily temperature was calculated.

Adults of the black garbage fly, Ophyra aenescens (Wiedemann), were found to be present during the second trial (13-17 June). The number of $O$. aenescens adults per house fly adult in each collection was used to estimate the number of $O$. aenescens.

Survival rates and average daily temperatures $\left({ }^{\circ} \mathrm{C}\right)$ from both studies were pooled and analyzed with a weighted multiple regression using reciprocals of the variances and covariances of the survival estimates as weighting factors. The model used was: $\hat{S} i=b_{0}+b_{1} X_{1}+b_{2} X_{2}$, where $X_{1}=$ average daily temperature $\left({ }^{\circ} \mathrm{C}\right), X_{2}=1$ if the observation came from the first study and $X_{2}=0$ if from the second. The value $b_{2}$ is an estimate of the average difference in daily survival estimates between the two experiments after accounting for the effects of temperature. An $F$ test was used to determine if the rate of change of survival with respect to temperature $\left(b_{1}\right)$ was homogenous among experiments.

\section{Results and Discussion}

Evaluation of Marking and Sampling Methods. The marking and sampling methods used met the assumptions of the Jolly-Seber model. There were no significant differences among the number of flies $1,3,5$, and 7 days old captured with the baited jug-traps $(F=0.84 ; \mathrm{df}=3,21 ; P=0.50)$. The arithmetic mean $( \pm \mathrm{SE})$ number of flies of each age captured (per 500 flies in $1-\mathrm{m}^{3}$ cages) was 90.4 $( \pm 12.34), 88.1( \pm 6.54), 92.6( \pm 10.80)$, and 76.6 ( \pm 9.43 ), for 1-, 3-, 5-, and 7-day-old flies, respectively. Males and females were caught in equal proportions (1,386 males/1,396 females). Marking did not affect capture; equal proportions of marked and unmarked flies were captured with the traps. The number of flies trapped and the proportion 
Table 1. Summary of recapture statistics, population size, and survival estimates for the house fly in a high-rise caged-layer poultry house

\begin{tabular}{|c|c|c|c|c|c|c|c|c|c|c|}
\hline Date & $i$ & $n_{\mathrm{i}}$ & $m_{i}$ & $R_{i}$ & $r_{i}$ & $\hat{N}_{i}( \pm \mathrm{SE})$ & $\hat{M}_{i}( \pm \mathrm{SE})$ & $\hat{U}_{\mathrm{i}}( \pm \mathrm{SE})$ & $\hat{S_{i}}( \pm \mathrm{SE})$ & $\begin{array}{c}\text { Correlation } \\
\left(\hat{S}_{i}, \hat{S}_{i+1}\right)\end{array}$ \\
\hline 25 May & 1 & - & - & 21,796 & 2,671 & - & - & - & $0.48(0.02)$ & -0.26 \\
\hline $26 \mathrm{May}$ & 2 & 2,481 & 1,779 & 18,788 & 1,892 & $14,834(1,176)$ & $10,639(341)$ & $4,197(1,064)$ & $0.51(0.02)$ & \\
\hline 27 May & 3 & 2,407 & 2,125 & 12,288 & 679 & $15,916(1,189)$ & $14,051(634)$ & $1,864 \quad(952)$ & - & \\
\hline 28 May & 4 & 1,652 & 1,338 & 0 & 0 & - & - & - & - & \\
\hline $\bar{x}$ & & & & & & $15,375 \quad(836)$ & $12,344(360)$ & $3,031 \quad(721)$ & $0.50(0.01)$ & \\
\hline 14 June & 1 & - & - & 8,738 & 692 & - & - & - & $0.49(0.03)$ & -0.32 \\
\hline 15 June & 2 & 1,298 & 464 & 9,445 & 567 & $11,923(2,484)$ & $4,262(289)$ & $7,661(2,405)$ & $0.30(0.02)$ & -0.26 \\
\hline 16 June & 3 & 1,389 & 561 & 10,054 & 697 & $9,746(1,640)$ & $3,936(246)$ & $5,810(1,565)$ & $0.20(0.01)$ & \\
\hline 17 June & 4 & 1,765 & 777 & 10,494 & 852 & $6,074 \quad(685)$ & $2,674(159)$ & $3,400 \quad(616)$ & - & \\
\hline 18 June & 5 & 2,087 & 1,006 & 0 & 0 & - & - & - & - & \\
\hline $\bar{x}$ & & & & & & $9,248(1,018)$ & $3,224(137)$ & $5,623 \quad(978)$ & $0.33(0.01)$ & \\
\hline 4 July & 1 & - & - & 10,098 & 774 & - & - & - & $0.19(0.01)$ & -0.14 \\
\hline 5 July & 2 & 1,567 & 623 & 10,230 & 1,217 & $4,670 \quad(467)$ & $1,892(103)$ & $2,867 \quad(419)$ & $0.36(0.02)$ & -0.23 \\
\hline 6 July & 3 & 1,974 & 1,118 & 9,746 & 807 & $7,305 \quad(721)$ & $4,137(209)$ & $3,187 \quad(640)$ & $0.38(0.02)$ & \\
\hline 7 July & 4 & 1,262 & 842 & 8,133 & 440 & $7,218(1,160)$ & $4,816(322)$ & $2,402(1,067)$ & - & \\
\hline 8 July & 5 & 949 & 655 & 0 & 0 & - & - & - & - & \\
\hline $\bar{x}$ & & & & & & $6,428 \quad(481)$ & $3,615(132)$ & $2,812 \quad(437)$ & $0.31(0.01)$ & \\
\hline 25 July & 1 & - & - & 10,252 & 1,858 & - & - & - & $0.66(0.02)$ & -0.37 \\
\hline 26 July & 2 & 4,079 & 1,114 & 9,812 & 1,303 & $24,593(2,231)$ & $6,717(240)$ & $17,887(2,148)$ & $0.41(0.02)$ & -0.27 \\
\hline 27 July & 3 & 4,301 & 1,494 & 9,262 & 1,047 & $18,384(1,480)$ & $6,386(242)$ & $11,988(1,383)$ & $0.49(0.02)$ & \\
\hline 28 July & 4 & 3,417 & 1,236 & 8,718 & 558 & $19,139(2,457)$ & $6,923(371)$ & $12,216(2,328)$ & - & \\
\hline 29 July & 5 & 2,631 & 922 & 0 & 0 & - & - & - & - & \\
\hline $\bar{x}$ & & & & & & $20,706(1,211)$ & $6,675(168)$ & $14,030(1,152)$ & $0.52(0.01)$ & \\
\hline 17 Aug. & 1 & - & - & 9,834 & 1,321 & - & - & - & $0.55(0.02)$ & -0.33 \\
\hline 18 Aug. & 2 & 1,369 & 790 & 10,010 & 1,157 & $9,330(1,044)$ & $5,384(226)$ & $3,949 \quad(981)$ & $0.45(0.02)$ & -0.31 \\
\hline 19 Aug. & 3 & 1,652 & 1,141 & 9,416 & 961 & $9,412 \quad(951)$ & $6,501(372)$ & $2,911 \quad(799)$ & $0.34(0.01)$ & \\
\hline 20 Aug. & 4 & 1,799 & 1,223 & 9,248 & 703 & $7,314 \quad(758)$ & $4,972(253)$ & $2,342 \quad(671)$ & - & \\
\hline 21 Aug. & 5 & 1,489 & 988 & 0 & 0 & - & - & - & - & \\
\hline $\bar{x}$ & & & & & & $8,685 \quad(534)$ & $5,619(168)$ & $3,066 \quad(477)$ & $0.44(0.01)$ & \\
\hline 10 Sept. & 1 & - & - & 10,318 & 1,275 & - & - & - & $0.29(0.02)$ & -0.21 \\
\hline 11 Sept. & 2 & 1,895 & 1,117 & 9,944 & 825 & $5,126 \quad(449)$ & $3,021(158)$ & $2,105 \quad(376)$ & $0.20(0.01)$ & -0.20 \\
\hline 12 Sept. & 3 & 1,683 & 848 & 10,098 & 920 & 4,624 & $2,230(130)$ & 2,294 & $0.24(0.01)$ & \\
\hline 13 Sept. & 4 & 1,485 & 822 & 9,812 & 1,137 & $5,118 \quad(467)$ & $2,833(136)$ & $2,285 \quad(412)$ & - & \\
\hline 14 Sept. & 5 & 2,266 & 1,370 & 0 & 0 & - & - & - & - & \\
\hline $\bar{x}$ & & & & & & $4,966 \quad(261)$ & $2,728 \quad(82)$ & 2,228 & $0.24(0.01)$ & \\
\hline
\end{tabular}

that were marked in each trial with Calco red, Calco violet, Calco green, and phenophthalein were $509,0.48\left(\chi^{2}=0.52 ; \mathrm{df}=1 ; P=0.45\right) ; 325,0.54$ $\left(\chi^{2}=1.12 ; \mathrm{df}=1 ; P=0.25\right) ; 427,0.54\left(\chi^{2}=1.12\right.$; $\mathrm{df}=1 ; P=0.25)$; and 268, $0.50\left(\chi^{2}=0.0 ; \mathrm{df}=1\right.$; $P=1.0$ ), respectively.

Dyes had no effect on mortality $(F=1.26$; df $=$ $4,20 ; P=0.30)$. The arithmetic mean $( \pm S E)$ of the number of dead flies 5 days posttreatment (five cages per dye, 25 male and 25 female flies per cage) were as follows: no dye, 2.2 (0.37); Calco red, $3.4(0.40)$; Calco violet, 1.4 (0.93); Calco green, $2.0(0.71)$; and phenolphthalein, $2.2(0.66)$. No mark loss was observed after a week.

Multisample, Single-recapture Census. The results of the multisample, single-recapture experiment conducted in the high-rise house are presented in Table 1. Estimates of population numbers $\left(\hat{N}_{f}\right)$, daily survival $\left(\hat{S}_{i}\right)$, numbers of marked flies $\left(\hat{M}_{i}\right)$, standard errors due to estimation of the parameters, and the correlation between survival estimates on successive days are shown. $\hat{N}_{i}$ is an estimate of the total number of flies present on a given day and is composed of both marked and unmarked flies. Therefore, the number of un- marked flies $\left(\hat{U}_{t}\right)$ present on a given day can be calculated as $\hat{U}_{i}=\hat{N}_{i}-\hat{M}_{i}$ with variance $\left(\hat{U}_{i}\right)=$ $\operatorname{Var}\left(\hat{N}_{i}\right)+\operatorname{Var}\left(\hat{M}_{i}\right)-2$ covariance $\left(\hat{N}_{i}, \hat{M}_{i}\right)$. The covariance of $\left(\hat{N}_{i}, \hat{M}_{i}\right)$ is approximately equal to $\left(n_{i} / m_{i}\right) \operatorname{Var}\left(\hat{M}_{i}\right)$ where $n_{i}=$ total number of flies in the sample on day $i$, and $m_{i}=$ number of marked flies caught on day $i$ (K. H. Pollock, personal communication). The unmarked flies represent the native flies since all marked flies were introduced. $R_{\mathrm{i}}=$ number of marked flies released on day $i$ and $r_{i}=$ number of $R_{i}$ captured.

Although large numbers of marked flies were released daily, $\hat{N}_{i}$ showed little tendency to increase within a week. This could have been due in part to the loss of marked flies due to dispersal and mortality, as well as the large number of flies removed by the traps. Estimates of $\hat{N}_{i}, \hat{M}_{i}, \hat{U}_{i}$, and $\hat{S}_{i}$ were averaged within weeks to allow comparisons between weeks, and the standard errors of the averaged estimates were calculated as a linear function of the individual within-week variances of the estimates. The averaged estimates are shown in Table 1 . There was a small peak in numbers in mid-June and a larger peak in late July. The population declined through August and September. 
Table 2. Summary of recapture statistics, population size, and survival estimates for the house fly in a narrow eaged-layer poultry house

\begin{tabular}{|c|c|c|c|c|c|c|c|c|c|c|c|}
\hline Date & $i$ & $n_{\mathrm{i}}$ & $m_{i}$ & $R_{i}$ & $r_{i}$ & $\hat{N}_{i}( \pm$ & SE) & $\hat{M}_{i}( \pm \mathrm{SE})$ & $\hat{S_{i}}( \pm \mathrm{SE})$ & $\begin{array}{c}\text { Pro- } \\
\text { portion } \\
\text { Ophyra } \\
\text { in } \\
\text { sample }\end{array}$ & $\begin{array}{c}\text { Total no. } \\
\text { Ophyra } \pm \mathrm{SE}\end{array}$ \\
\hline 20 May & 1 & 295 & 0 & 295 & 197 & 541 & $(38.5)$ & - & $0.94(0.03)^{a b}$ & & \\
\hline 22 May & 3 & 266 & 129 & 218 & 110 & 378 & $(13.2)$ & $263 \quad(14.6)$ & $0.74(0.03)^{a c}$ & & \\
\hline 24 May & 5 & 320 & 162 & 320 & 171 & 505 & $(46.6)$ & $192 \quad(5.3)$ & $0.88(0.04)^{a}$ & & \\
\hline 26 May & 7 & 305 & 164 & 305 & 64 & - & & $272 \quad(23.5)$ & - & & \\
\hline 28 May & 9 & 236 & 87 & 236 & & - & & - & - & & \\
\hline 13 June & 1 & 931 & 0 & 931 & 90 & - & & - & $0.72(0.24)^{a}$ & & \\
\hline 15 June & 3 & 578 & 84 & 525 & 7 & 3,259 ( & $1,567.2)$ & $479(276.1)$ & - & 0.12 & 389 (187) \\
\hline 17 June & 5 & 513 & 13 & 513 & & 1 & & - & - & & \\
\hline 26 June & 1 & 3,011 & 0 & 2,626 & 449 & - & & - & $0.99(0.11)$ & & \\
\hline 27 June & 2 & 1,576 & 234 & 1,222 & 100 & 17,614 & $2,114.3)$ & $2,625(276.6)$ & - & 0.07 & $1,212(145)$ \\
\hline 28 June & 3 & 2,341 & 315 & 2,341 & & - & & - & - & & \\
\hline 3 July & 1 & 2,330 & 0 & 1,978 & 551 & - & & - & $0.97(0.08)$ & & \\
\hline 4 July & 2 & 1,392 & 294 & 913 & 143 & 9,091 & (856.3) & $1,925 \quad(56.4)$ & - & 0.89 & $8,070(760)$ \\
\hline 5 July & 3 & 1,403 & 400 & 1,403 & & - & & - & - & & \\
\hline 17 July & 1 & 1,066 & 0 & 708 & 185 & - & & - & $0.74(0.08)$ & & \\
\hline 18 July & 2 & 991 & 121 & 986 & 156 & 4,255 & $(547.3)$ & $523(54.9)$ & - & 0.87 & $3,662(471)$ \\
\hline 19 July & 3 & 3 & 818 & 220 & & - & & - & - & & \\
\hline 24 July & 1 & 1,624 & 0 & 1,624 & 537 & - & & - & $0.80(0.05)$ & & \\
\hline 25 July & 2 & 1,281 & 324 & 1,281 & 277 & 5,153 & $(379.2)$ & $1,306(179.3)$ & - & 0.58 & $2,871(219)$ \\
\hline 26 July & 3 & 1,479 & 490 & 1,479 & & - & & - & - & & \\
\hline 31 July & 1 & 871 & 0 & 871 & 267 & - & & - & $0.63(0.05)$ & & \\
\hline 1 Aug. & 2 & 704 & 185 & 704 & 158 & 2,079 & (196.7) & $549(43.7)$ & - & 1.21 & $2,506(237)$ \\
\hline 2 Aug. & 3 & 679 & 240 & 679 & & - & & - & - & & \\
\hline 7 Aug. & 1 & 273 & 0 & 273 & 76 & - & & - & $0.54(0.08)$ & & \\
\hline 8 Aug. & 2 & 193 & 53 & 193 & 46 & 532 & $(90.8)$ & $148(21.3)$ & - & 5.97 & $3,171(542)$ \\
\hline 9 Aug. & 3 & 219 & 69 & 219 & & - & & - & - & & \\
\hline
\end{tabular}

${ }^{a}$ Original estimates of survival for 2-day intervals were converted to estimates of survival for 1-day intervals.

${ }^{b}$ Correlation between $S_{i}$, and $S_{i+1}=-0.79$.

${ }^{c}$ Correlation between $S_{i}$, and $S_{i+1}=-0.10$.

Numbers of house flies were low in this highrise house, and ranged from 1,864 to 17,887 (1.211.5 flies per $\mathrm{m}^{2}$ ) with a mean of 5,132 (3.3 flies per $\mathrm{m}^{2}$ ). The estimates of the number of unmarked flies were lower than expected; however, the house had a history of low fly densities as a result of liquid manure condition (personal observation). Numbers of house flies may be higher in other situations. In a different type of housing, Beck (1982) used the Petersen model to estimate numbers of house flies in two shallow-pit, caged-layer poultry houses (ca. $110 \mathrm{~m}^{2}$ area) in Virginia. Beck (1982) sampled the house fly populations $1 \mathrm{~h}$ after release of marked laboratory flies to minimize loss of marked flies. Estimates of the number of wild house flies ranged from 4,457 to 44,369 (40.5-403.4 flies per $\mathrm{m}^{2}$ ) and averaged 17,213 (156.5 flies per $\mathrm{m}^{2}$ ); however, the two highest estimates were based on the lowest percent recapture of marked flies $(<0.2 \%, 7$ marked flies each in samples of 88 and 113 flies). If these are ignored, the number of house flies ranged from 4,457 to 22,480 (40.5-204.4 flies per $\mathrm{m}^{2}$ ) with a mean of $10,574\left(96.1\right.$ flies per $\left.\mathrm{m}^{2}\right)$ flies.

Newly released flies may have a higher level of activity than flies already in the population (Southwood 1978) and might have a higher probability of capture. This would cause $\hat{M}_{i}$ and $\hat{N}_{i}$ to be underestimated, but $S_{i}$ would be less affected (Cormack 1972). Effects due to short-term increases in capture probabilities could be reduced by releasing and recapturing on alternate days.

Adult survival rates were low in June and early July, increased in late July and August, and were low in September. The survival estimates measure the proportion of marked flies that were not lost during the sampling interval and are corrected for losses due to capture (Jolly 1965). Since the highrise house was not enclosed, losses from the population could have been due to both natural mortality (i.e., excluding death due to trapping) and permanent emigration.

Precision of the estimates was high. The average coefficient of variation of the daily estimates of $\hat{N}_{i}, \hat{M}_{i}, \hat{U}_{i}$, and $\hat{S}_{i}$ were $0.11,0.05,0.24$ and 0.05 , respectively. The mean CV's of the averaged estimates of $\hat{N}_{i}, \hat{M}_{i}, \hat{U}_{i}$, and $\hat{S}_{i}$ were $0.07,0.03,0.15$, and 0.03 , respectively. The average proportion of marked flies in the total population $\left(\hat{M}_{i} / \hat{N}_{i}\right)$, proportion of marked flies captured $\left(m_{i} / \hat{M}_{i}\right)$, and proportion of the released flies recaptured $\left(r_{i} / R_{i}\right)$ were $0.53,0.54$, and 0.10 , respectively, and were responsible for the high precision of the estimates.

A fourth assumption inherent in this method is that the number of flies released each day $\left(R_{i}\right)$ was known exactly. This quantity can only be estimat- 
ed in experiments of this size. Therefore, simulations were used to detect the effect of departure from this assumption on the estimates $\hat{N}_{i}$ and $\hat{S}_{i}$. The data in Table 1 were reanalyzed substituting values of $R_{i}$ ranging from -50 to $+50 \%$ of the original values. The resulting bias in $\hat{N}_{i}$ was 0.78 (percent error in $R_{i}$ ) (i.e., if $R_{i}$ was consistently overestimated by $10 \%, \hat{N}_{\mathrm{i}}$ would have been overestimated by $7.8 \%$ ). Similarly, the bias in $\hat{S}_{i}$ was -0.23 (percent error $R_{i}$ ); if $R_{i}$ was overestimated by $10 \%, \hat{S}_{i}$ would have been underestimated by $2.3 \%$.

Marked and unmarked flies were recaptured in similar locations within the house. Of all unmarked flies captured, $29.4 \%$ were caught along the sides above the walkway, $4.2 \%$ were caught in the center above the walkway, $40.4 \%$ along the sides below the walkway, and $26.0 \%$ along the center below the walkway. Of the marked flies captured, $20.0,2.1,54.9$, and $23.0 \%$ were caught in these respective areas. A similar distribution of flies within high-rise houses was observed by Burg \& Axtell (1984). In conducting a census of this type, it is important to sample throughout the entire house to obtain a representative sample of the marked and unmarked fly populations.

Multisample, Multirecapture Census. The results of the multisample, multirecapture experiment in the enclosed narrow poultry house are shown in Table 2. Since this experiment involved live captures and releases of native flies only, $N_{\text {i }}$ and $M_{i}$ are presented; $U_{i}$ has no biological significance. Numbers of house flies increased from late May until early June, then decreased after the end of June and few flies were left by early August. This decline in numbers was associated with increasing liquification of the manure and preceded a rise in the number of adult $O$. aenescens, the larvae of which are predaceous on house fly immatures, and probably acted to reduce house fly breeding.

This experiment was also quite precise; the average coefficient of variation was $0.14,0.12$, and 0.13 for $\hat{N}_{i}, \hat{M}_{i}$, and $\hat{S}_{i}$, respectively. The average proportion of marked flies in the total population $\left(\hat{M}_{i} / \hat{N}_{i}\right)$, proportion of marked flies captured $\left(m_{i} /\right.$ $\left.\hat{M}_{i}\right)$ and proportion of released flies recaptured $\left(r_{i} /\right.$ $R_{i}$ ) were $0.30,0.35$, and 0.26 , respectively. These were smaller than in the previous experiments due to less sampling intensity and are reflected in the slightly lower precision of this experiment.

The assumptions of the Jolly-Seber method were probably met because marked and unmarked flies would be equally likely caught with a sweep net. The fluorescent powders have not affected mortality in a variety of Diptera (Chamberlain et al. 1977, Nelson et al. 1978, Lillie et al. 1981), and they adhered to the adult flies for at least 1 week in cage tests (personal observation, based on 500 flies per $1-\mathrm{m}^{3}$ cage).

The survival rates obtained during the first two trials were for 2-day intervals, and were converted

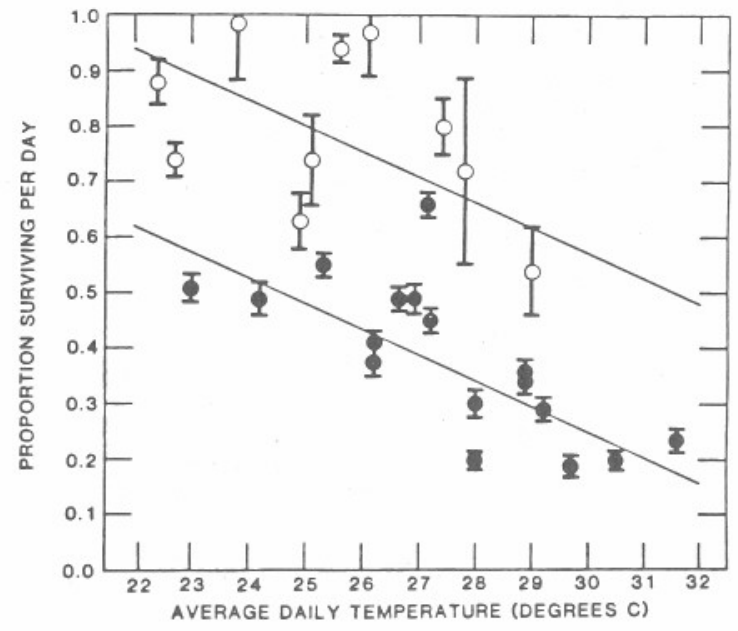

Fig. 1. Relationship between average daily temperature and daily survival of the house fly. estimates from an open-sided, high-rise poultry house; $\bigcirc$, a screened narrow poultry house. The lines are the regression equation $y=1.941-0.046 \mathrm{Temp}\left({ }^{\circ} \mathrm{C}\right)-0.319 x$. Upper line is relationship with no emigration or predation $(x=0$, screened narrow house); lower line is relationship with emigration and predation $(x=1$, highrise house). Bars are $\mathrm{SE}$ of the estimates.

to daily survival rates by taking the square root of each. The variances and covariances of the converted estimates were calculated using the delta method (Seber 1980). Adult survival was highest from late June through July and declined in August. Daily survival rates were higher $(0.54-0.99)$ than in the previous experiment $(0.19-0.66)$ and were similar to those reported by Imai (1984) and Kristiansen \& Skovmand (1985), 0.63-0.84 and 0.54-0.92, respectively.

The relationships between daily survival rate and temperature for both experiments are shown in Fig. 1. The change in survival rates with respect to temperature was homogenous between experiments $(F=0.98$; $\mathrm{df}=1,23 ; P>0.30)$, which justifies the use of pooled data from both experiments. The regression equation was: $\hat{S}_{i}=1.941-$ $0.046 \mathrm{Temp}\left({ }^{\circ} \mathrm{C}\right)-0.319 x \quad\left(r^{2}=0.89 ; \mathrm{SE}\left(b_{0}\right)=\right.$ $\left.0.0342, \mathrm{SE}\left(b_{1}\right)=0.0075, \mathrm{SE}\left(b_{2}\right)=0.0500 ; P=0.01\right)$ where $x=1$ if the observation came from the first study and $x=0$ if it came from the second. The value -0.319 is, therefore, the average difference in daily survival estimates due to dispersal and predation of adult flies in the first experiment, after the effects of temperature are accounted for.

The range in survival rate of marked flies and the relationship between average daily temperature and daily survival indicate that unless sampling is conducted over a very short period of time $(\leq 1 \mathrm{~h})$, a closed model such as the Petersen model is inappropriate, and an open model should be used to estimate numbers of house flies. The multisample, single-recapture census is a valuable tool for estimating numbers of house flies and other 
highly mobile insects when sampling must be conducted with traps that kill the insect. In addition to allowing for losses over the sample period, its advantages are as follows: 1) marking can be completed in the laboratory, not the field; 2) captured insects can be examined for marks in the laboratory and do not have to be released again; 3) a large number of marked individuals can be established in the population; and 4) sample sizes can be large without great amounts of effort.

\section{Acknowledgment}

The advice and suggestions of K. H. Pollock (Dep. of Statistics, North Carolina State Univ.) are gratefully acknowledged. This is Paper No. 10146 of the Journal Series of the N.C. Agric. Res. Serv., Raleigh, NC 276957601 .

\section{References Cited}

Bailey, N. J. J. 1952. Improvements in the interpretation of recapture data. J. Anim. Ecol. 21: 120-127.

Beck, A. F. 1982. An analysis of muscoid fly monitoring techniques with emphasis on the role of visual perception. Ph.D. dissertation, Virginia Polytechnic Institute and State Univ., Blacksburg.

Begon, M. 1979. Investigating animal abundance: capture-recapture for biologists. University Park, Baltimore, Md.

Burg, J. G. \& R. C. Axtell. 1984. Monitoring house fly (Musca domestica) (Diptera: Muscidae) populations in caged-layer poultry houses using a baited jug-trap. Environ. Entomol. 13: 1083-1090.

Chamberlain, W. F., J. A. Miller, M. O. Pickens, A. R. Gingrich \& C. I. Edwards. 1977. Marking horn flies with fluorescent dyes and other materials. J. Econ. Entomol. 70: 586-588.

Cormack, R. M. 1972. The logic of capture-recapture estimates. Biometrics 28: 337-343.

Fletcher, B. S., E. Kapatos \& T. R. E. Southwood. 1981. A modification of the Lincoln index for estimating the population densities of mobile insects. Ecol. Entomol. 6: 397-400.

Imai, C. 1984. Population dynamics of house flies, Musca domestica, on experimentally accumulated refuse. Res. Popul. Ecol. 26: 353-362.

Jolly, G. M. 1965. Explicit estimates from capture- recapture data with both death and immigrationstochastic model. Biometrika 52: 225-247.

Kristiansen, K. \& O. Skovmand. 1985. A method for the study of population size and survival rate of houseflies. Entomol. Exp. Appl. 38: 145-150.

Lillie, T. H., R. H. Jones \& W. C. Marquardt. 1981. Micronized fluorescent dusts for marking Culicoides variipennis adults. Mosq. News 41: 356-358.

Lysyk, T. J. \& R. C. Axtell. 1986. Movement and distribution of house flies (Diptera: Muscidae) between habitats in two livestock farms. J. Econ. Entomol. 79: 988-993.

MeDonald, I. C., D. A. Johnson, C. Niekel, P. Evenson \& D. Birkenmeyer. 1983. House fly (Diptera: Muscidae) genetics: field studies of males from a maleproducing strain. Ann. Entomol. Soc. Am. 76: 333338.

Murvosh, C. M. \& C. W. Thaggard. 1966. Ecological studies of the house fly. Ann. Entomol. Soc. Am. 59: 533-547.

Nelson, R. L., M. M. Milby, W. C. Reeves \& P. E. M. Fine. 1978. Estimates of survival, population size and emergence of Culex tarsalis at an isolated site. Ann. Entomol. Soc. Am. 71: 801-808.

Peffly, R. L. \& G. C. LaBrecque. 1956. Marking and trapping studies on dispersal and abundance of Egyptian house flies. J. Econ. Entomol. 49: 214-217.

Pollock, K. H. 1981. Capture-recapture models: a review of current methods, assumptions and experimental design, pp. 426-435. In C. J. Ralph \& J. M. Scott [eds.], Estimating the numbers of terrestrial birds. Studies in Avian Biology 6, Cooper Ornithological Society.

Pospisil, Y. \& Y. Bogac. 1982. Comparison of methods for estimating population density of the house fly (Musca domestica, Diptera: Muscidae) at cattle farms. Ekologiya 4: 77-82.

Quarterman, K. D., W. Mathis \& J. W. Kilpatrick. 1954. Urban fly dispersal in the area of Savannah, Georgia. J. Econ. Entomol. 47: 405-412.

Seber, G. A. F. 1962. The multi-sample single recapture census. Biometrika 48: 339-349.

1980. The estimation of animal abundance and related parameters, 2nd ed. Griffin, London.

Southwood, T. R. E. 1978. Ecological methods with particular reference to the study of insect populations, 2nd ed. Chapman \& Hall, London.

Received for publication 9 January 1986; accepted 31 March 1986. 